\title{
Rigorous Formulation of Duality in Gravitational Theories
}

\author{
Roldão da Rocha ${ }^{(1)}$ and Waldyr A. Rodrigues Jr. ${ }^{(2)}$ \\ ${ }^{(1)}$ Centro de Matemática, Computação e Cognição \\ Universidade Federal do ABC, 09210-170, Santo André, SP, Brazil \\ roldao.rocha@ufabc.edu.br \\ ${ }^{(2)}$ Institute of Mathematics, Statistics and Scientific Computation \\ IMECC-UNICAMP CP 6065 \\ 13083-859 Campinas, SP, Brazil \\ walrod@ime.unicamp.br, walrod@mpc.com.br
}

November 17, 2018

\begin{abstract}
In this paper we evince a rigorous formulation of duality in gravitational theories where an Einstein like equation is valid, by providing the conditions under which $\star \mathcal{T}^{\alpha}$ and $\star \mathcal{R}_{\beta}^{\alpha}$ may be considered as the torsion and curvature 2-forms associated with a connection $D^{\prime}$, part of a Riemann-Cartan structure $\left(M, \mathbf{g}^{\prime}, D^{\prime}\right)$, in the cases $g^{\prime}=g$ and $g^{\prime} \neq g$, once $\mathcal{T}^{\alpha}$ and $\mathcal{R}_{\beta}^{\alpha}$ are the torsion and curvature 2 -forms associated with a connection $D$ part of a Riemann-Cartan structure $(M, g, D)$. A new form for the Einstein equation involving the dual of the Riemann tensor of $D$ is also provided, and the result is compared with others appearing in the literature.
\end{abstract}

\section{Introduction}

There has been a number of papers trying to put in evidence a possible analogy between electromagnetism and gravitation, in order to elicit a gravitational analogue for the magnetic monopole that appears in the generalized Maxwell equations with magnetic and electric current:1. Among the old ones we quot 2 [ [9, 17, 18]. Ten years ago Nieto [19] developed an analogue of S-duality 3 for linearized gravity in $(3+1)$ dimensions (see, also 12, 20, 13]) and generalizations of that idea of duality for gravitational theories in more dimensions appear, e.g., in [1, 2, 3, 5, 6, 7, 11. In particular for the case of gravity in $(3+1)$ dimensions a set of equations has been proposed for Einstein equations, Bianchi identities, and their duals, although mainly used in the linear approximation. The main aim of this note is to derive exact equations that must be satisfied by the dual Einstein equations and for the duals of the torsion and curvature 2-forms of a general Riemann-Cartan structure $(M, \mathbf{g}, D)$. We study in which conditions the dualized objects realize a Riemann-Cartan structure $\left(M, \boldsymbol{g}, D^{\prime}\right)$, or even a $\left(M, \mathbf{g}^{\prime}, D^{\prime}\right)$ one. In so doing we find that the correct field equations for a dual theory (in a precise mathematical sense defined below), are at variance with ones proposed in some of the above mentioned papers. In so doing we hope that the present note be useful for those pursuing the interesting ideas of duality in gravitational theories.

The paper, which uses an intrinsic formulation of the theories presented, is organized as follows. In Section 2 we present some necessary preliminaries that serve, besides the proposal of introducing our notation, also the one of presenting what it is understood here by a Riemann-Cartan gravitational theory. In this Section we review also the Bianchi identities for the torsion and curvature 2 -forms $\mathcal{T}^{\alpha}$ and $\mathcal{R}_{\beta}^{\alpha}$ of $(M, \mathbf{g}, D)$ in intrinsic

\footnotetext{
${ }^{1}$ In such theory, see, e.g., $15 \sqrt{25}$ which uses two potentials, the electric and magnetic currents are phenomenological, i.e., the magnetic current is not a result of a $U(1)$ gauge theory formulated in a nontrivial base spacetime. So, in the theory which uses two potentials there are no Dirac strings at all. Unfortunately, this result is sometimes overlooked in presentations of the monopole theory and in the proposed gravitational analogies of that concept.

${ }^{2}$ One of the motivations of $[9]$ was eventually to obtain a quantization of mass.

${ }^{3}$ Duality and S-duality have been also studied extensively in non abelian gauge theories, see, e.g. [21] 16] 26] and references therein.
} 
and component forms, because those formulas for a Riemann-Cartan theory are not well known as they deserve to be, and sometimes concealed from the formalism. In Section 3 we introduce the Ricci 1-form fields $\mathcal{R}^{\mu}$ and the Einstein 1-forms fields $\mathcal{G}^{\mu}[23$, and further prove a Proposition containing a formula that relates the dual $\star \mathcal{R}^{\mu}$ of $\mathcal{R}^{\mu}$ to a sum, involving the dual of the Riemann tensor and an important formula for the dual $\star \mathcal{G}^{\mu}$ of $\mathcal{G}^{\mu}$, that permits us to write Einstein equations in a suggestive way concerning duality structures. In $\stackrel{g}{\text { Section }} 4$ we provide the correct dual of Einstein equation in Riemann-Cartan theory. In Section 5 we delve into the formalism under which conditions $\star \mathcal{T}^{\alpha}$ and $\star \mathcal{R}_{\beta}^{\alpha}$ may be considered as the torsion and curvature 2 -forms associated with a connection $D^{\prime}$ part of a Riemann-Cartan structure $\left(M, \mathbf{g}, D^{\prime}\right)$. Our result is then compared in Section 6 with the ones, e.g., in [1], which are then commented and analyzed in the present context. In Section 7 we study the same problem as in Section 5 but this time asking the conditions under which $\underset{\mathrm{g}}{\star} \mathcal{T}^{\alpha}$ and $\underset{\mathrm{g}}{\star \mathcal{R}_{\beta}} \underset{\mathrm{s}}{\alpha}$ may be considered as the torsion and curvature 2-forms associated to a connection $D^{\prime}$ part of a Riemann-Cartan structure $\left(M, \mathbf{g}^{\prime}, D^{\prime}\right)$ with $\mathbf{g}^{\prime} \neq \mathbf{g}$. In Section 8 we present our conclusions. The paper contains some Appendices reviewing the definition of the exterior covariant derivative of indexed form fields, the decomposition of the Riemann and Ricci tensors of a general Riemann-Cartan structure $(M, \boldsymbol{g}, D)$, together with their respective similars for a Lorentzian structure $(M, \stackrel{\circ}{\mathrm{g}}, \stackrel{\circ}{D})$, needed to perceive some statements in the main text. There is also an Appendix containing a collection of identities involving the contraction of differential forms and Hodge duals used in the derivations hereon.

\section{Some Necessary Preliminaries}

We start this Section by recalling some germane facts concerning the Riemann-Cartan structures and a particular and outstanding case of those structures, the Lorentzian one, which serves for the purpose of fixing our notations, besides other relevant properties and prominent applications. In what follows a general RiemannCartan structure will be denoted by $(M, \mathbf{g}, D)$. Here $M$ is a 4-dimensional Hausdorff, paracompact, connected, and noncompact manifold, $\boldsymbol{g} \in \sec T_{2}^{0} M$ a metric tensor field of signature (1,3), $D$ is a connection on $M$. Also the connection $D$ is metric compatible, i.e., $D \boldsymbol{g}=0$ and, moreover, for a general Riemann-Cartan structure the torsion and curvature tensors 4 of $D$ - denoted by $\mathcal{T}$ and $\mathbf{R}-$ are non null. When $\mathcal{T}=0$ and $\mathbf{R} \neq 0$, a Riemann-Cartan structure is called a Lorentzian structure and will be denoted by $(M, \mathbf{g}, D) \sqrt{5}$. When $\mathbf{R}=0$ a Lorentzian structure is called Minkowski structure. To present the definition of $\mathcal{T}$ and $\mathbf{R}$, and the conventions used in this paper, first the torsion and curvature operations are introduced.

Definition 1 Let $\mathbf{u}, \mathbf{v} \in \sec T M$. The torsion and curvature operations of a connection D, are respectively the mappings: $\tau: \sec T M \otimes \sec T M \rightarrow \sec T M$ and $\rho: \sec T M \otimes \sec T M \rightarrow \sec T M$ given by

$$
\begin{aligned}
\tau(\mathbf{u}, \mathbf{v}) & =\nabla_{\mathbf{u}} \mathbf{v}-\nabla_{\mathbf{v}} \mathbf{u}-[\mathbf{u}, \mathbf{v}], \\
\rho(\mathbf{u}, \mathbf{v}) & =\nabla_{\mathbf{u}} \nabla_{\mathbf{v}}-\nabla_{\mathbf{v}} \nabla_{\mathbf{u}}-\nabla_{[\mathbf{u}, \mathbf{v}]} .
\end{aligned}
$$

Definition 2 Let $\mathbf{u}, \mathbf{v}, \mathbf{w} \in \sec T M$ and $\alpha \in \sec \Lambda^{1} T^{*} M$. The torsion and curvature tensors of a connection $D$ are the mappings $\mathcal{T}: \sec \left(\Lambda^{1} T^{*} M \otimes T M \otimes T M\right) \rightarrow \mathbb{R}$ and $\mathbf{R}: \sec \left(T M \otimes \Lambda^{1} T^{*} M \otimes T M \otimes T M\right) \rightarrow \mathbb{R}$ given by

$$
\begin{aligned}
\mathcal{T}(\alpha, \mathbf{u}, \mathbf{v}) & =\alpha(\tau(\mathbf{u}, \mathbf{v})), \\
\mathbf{R}(\mathbf{w}, \alpha, \mathbf{u}, \mathbf{v}) & =\alpha(\rho(\mathbf{u}, \mathbf{v}) \mathbf{w}) .
\end{aligned}
$$

Given an arbitrary moving frame $\left\{\boldsymbol{e}_{\alpha}\right\}$ on $T M$, let $\left\{\theta^{\rho}\right\}$ be the dual frame of $\left\{\boldsymbol{e}_{\alpha}\right\}$ (i.e., $\left.\theta^{\rho}\left(\mathbf{e}_{\alpha}\right)=\delta_{\alpha}^{\rho}\right)$. Let also $\left\{\boldsymbol{e}^{\alpha}\right\}$ be the reciprocal basis of $\left\{\boldsymbol{e}_{\beta}\right\}$, i.e., $\mathbf{g}\left(\boldsymbol{e}^{\alpha}, \boldsymbol{e}_{\beta}\right)=\delta_{\beta}^{\alpha}$ and let $\left\{\theta_{\alpha}\right\}$ be the reciprocal basis of $\left\{\theta^{\rho}\right\}$, i.e., $\theta_{\alpha}\left(\mathbf{e}^{\beta}\right)=\delta_{\alpha}^{\beta}$. We write

$$
\left[\boldsymbol{e}_{\alpha}, \mathbf{e}_{\beta}\right]=c_{\alpha \beta}^{\rho} \mathbf{e}_{\rho}, \quad D \boldsymbol{e}_{\alpha} \mathbf{e}_{\beta}=L_{\alpha \beta}^{\rho} \mathbf{e}_{\rho},
$$

where $c_{\alpha \beta}^{\rho}$ are the structure coefficients of the frame $\left\{\mathbf{e}_{\alpha}\right\}$ and $L_{\alpha \beta}^{\rho}$ are the connection coefficients in this frame. Then, the components of the torsion and curvature tensors are given, respectively, by:

$$
\begin{gathered}
\mathcal{T}\left(\theta^{\alpha}, \mathbf{e}_{\alpha}, \mathbf{e}_{\beta}\right)=T_{\alpha \beta}^{\rho}=L_{\alpha \beta}^{\rho}-L_{\beta \alpha}^{\rho}-c_{\alpha \beta}^{\rho} \\
\mathbf{R}\left(\mathbf{e}_{\mu}, \theta^{\alpha}, \mathbf{e}_{\alpha}, \mathbf{e}_{\beta}\right)=R_{\mu}{ }^{\rho} \alpha \beta \\
=\mathbf{e}_{\alpha}\left(L_{\beta \mu}^{\rho}\right)-\mathbf{e}_{\beta}\left(L_{\alpha \mu}^{\rho}\right)+L_{\alpha \sigma}^{\rho} L_{\beta \mu}^{\sigma}-L_{\beta \sigma}^{\rho} L_{\alpha \mu}^{\sigma}-c_{\alpha \beta}^{\sigma} L_{\sigma \mu}^{\rho} .
\end{gathered}
$$

\footnotetext{
${ }^{4}$ For the conventions used for those tensors in this paper see the Appendix.

${ }^{5}$ The connection satisfying $\stackrel{\circ}{D} g=0$ and $\mathcal{T}=0$ is unique and is called the Levi-Civita connection of $g$.
} 
We can easily verify that defining

$$
R_{\mu \nu \alpha \beta}:=g_{\mu \rho} R_{\mu}{ }^{\rho} \alpha \beta
$$

it follows that

$$
R_{\mu \nu \alpha \beta}=R_{\nu \mu \alpha \beta}=R_{\mu \nu \beta \alpha} .
$$

Remark 3 When the torsion tensor of $D$ is null, besides the symmetries given in Eq.(8), also the symmetry

$$
R_{\mu \nu \alpha \beta}=R_{\beta \alpha \mu \nu}
$$

holds.

Now, taking into account Eq.(8) we introduce also a "physically equivalent" Riemann tensor $\boldsymbol{R}$ by

$$
\begin{aligned}
\boldsymbol{R} & =\frac{1}{4} R_{\mu \nu \alpha \beta} \theta^{\mu} \wedge \theta^{\nu} \otimes \theta^{\alpha} \wedge \theta^{\beta}=\frac{1}{4} R_{\alpha \beta}^{\mu \nu} \theta_{\mu} \wedge \theta_{\nu} \otimes \theta^{\alpha} \wedge \theta^{\beta} \\
& =\frac{1}{4} R_{\mu \nu}^{\alpha \beta} \theta^{\mu} \wedge \theta^{\nu} \otimes \theta_{\alpha} \wedge \theta_{\beta} .
\end{aligned}
$$

In addition,

$$
d \theta^{\rho}=-\frac{1}{2} c_{\alpha \beta}^{\rho} \theta^{\alpha} \wedge \theta^{\beta}, \quad D_{e_{\alpha}} \theta^{\rho}=-L_{\alpha \beta}^{\rho} \theta^{\beta}
$$

where $\omega_{\beta}^{\rho} \in \sec \Lambda^{1} T^{*} M$ are the connection 1-forms, $\mathcal{T}^{\rho} \in \sec \Lambda^{2} T^{*} M$ are the torsion 2-forms and $\mathcal{R}_{\beta}^{\rho} \in$ $\sec \Lambda^{2} T^{*} M$ are the curvature 2 -forms, given respectively by

$$
\omega_{\beta}^{\rho}=L_{\alpha \beta}^{\rho} \theta^{\alpha}, \quad \mathcal{T}^{\rho}=\frac{1}{2} T_{\alpha \beta}^{\rho} \theta^{\alpha} \wedge \theta^{\beta}, \quad \mathcal{R}_{\mu}^{\rho}=\frac{1}{2} R_{\mu}{ }^{\rho}{ }_{\alpha \beta} \theta^{\alpha} \wedge \theta^{\beta} .
$$

Multiplying Eqs.(6) by $\frac{1}{2} \theta^{\alpha} \wedge \theta^{\beta}$ and using Eqs.(11) and (12), the Cartan's structure equations are derived:

$$
\mathcal{T}^{\rho}=d \theta^{\rho}+\omega_{\beta}^{\rho} \wedge \theta^{\beta}, \quad \mathcal{R}_{\mu}^{\rho}=d \omega_{\mu}^{\rho}+\omega_{\beta}^{\rho} \wedge \omega_{\mu}^{\beta} .
$$

Definition 4 A Riemann-Cartan spacetime is a pentuple $\left(M, \mathbf{g}, D, \tau_{g}, \uparrow\right)$ where $(M, \boldsymbol{g}, D)$ is a Riemann-Cartan structure, and we suppose the existence of a global $\tau_{g} \in \sec \Lambda^{4} T^{*} M$ (which as well known defines an orientation for M). Moreover, $\uparrow$ denotes that the Riemann-Cartan structure is time oriented. See, e.g., 23, 22 for details.

Pentuples $\left(M, g, D, \tau_{g}, \uparrow\right)$ represent gravitational fields in the so called Riemann-Cartan theories. In the theory presented, e.g., in [14, the equations of motion are the Einstein equation,

$$
\mathbf{G}=\mathbf{T},
$$

where $\mathbf{G} \in \sec T_{2}^{0} M$ is the Einstein tensor, $\mathbf{T} \in \sec T_{2}^{0} M$ is the canonical energy-momentum tensor of the matter fields, and the algebraic identity

$$
\Upsilon_{\alpha \beta}=\mathbf{J}_{\alpha \beta},
$$

where the $\boldsymbol{\Upsilon}_{\alpha \beta} \in \sec \Lambda^{1} T^{*} M$ are such that their components are the so called modified torsion tensor components, and the $\star \mathbf{J}_{\alpha \beta} \in \sec \Lambda^{3} T^{*} M$ are the spin angular momentum densities of the matter field\&6. Also, the symbol $\underset{g}{\star}$ denotes the Hodge star operator associated to the metric $\mathbf{g}$.

Remark 5 It is crucial to observe that for a general Riemann-Cartan structure $\mathbf{G}=G_{\mu \nu} \theta^{\mu} \otimes \theta^{\nu}$ and $\mathbf{T}=$ $T_{\mu \nu} \theta^{\mu} \otimes \theta^{\nu}$ are not symmetric, i.e., $G_{\mu \nu} \neq G_{\nu \mu}$ and $T_{\mu \nu} \neq T_{\nu \mu}$. We recall that

$$
G_{\mu \nu}=R_{\mu \nu}-\frac{1}{2} g_{\mu \nu} R
$$

where $R_{\mu \nu}$ are the components of the Ricci tensor (which, as $G_{\mu \nu}$ are not symmetric)

$$
\text { Ricci }=R_{\mu \nu} \theta^{\mu} \otimes \theta^{\nu}:=R_{\mu}{ }^{\rho}{ }_{\rho \nu} \theta^{\mu} \otimes \theta^{\nu},
$$

and $R=g^{\mu \nu} R_{\mu} \rho_{\rho \nu}$ is the curvature scalar.

\footnotetext{
${ }^{6}$ The components of $\mathbf{J}_{\alpha \beta} \in \sec \Lambda^{1} T^{*} M$ are the standard (field theory) canonical spin angular momentum of the matter fields. In the Riemann-Cartan theory of [14], since Eq.(14) is an algebraic identity, it is possible to eliminate completely the torsion tensor from the theory and to write an Einstein equation involving the Einstein tensor of the Levi-Civita connection of $g$ (using the decomposition presented in Appendix B) and a metric energy-momentum tensor that is equivalent to the Belinfante symmetrization of the canonical energy-momentum tensor of the theory.
} 
It is also well known that in GRT a gravitational field generated by a given matter distribution (represented by a given energy-momentum tensor $\left.\stackrel{\circ}{\mathbf{T}} \in \sec T_{2}^{0} M\right)$ is represented by a pentuple $\left(M, \stackrel{\circ}{\mathrm{g}}, \stackrel{\circ}{D}, \tau_{\mathrm{g}}^{\circ}, \uparrow\right)$ 五 and the equation of motion (Einstein equation) is given by

$$
\stackrel{\circ}{\mathbf{G}}=\stackrel{\circ}{\mathbf{T}}
$$

and in this case the tensors $\stackrel{\mathbf{G}}{\text { and }} \stackrel{\circ}{\mathbf{T}}$ are symmetric.

Remark 6 In the Appendix we review how to write the Riemann curvature tensor (respectively the Einstein tensor) of a Riemann-Cartan structure $(M, \mathbf{g}, D)$ in terms of the Riemann curvature tensor (respectively the Einstein tensor) of a Lorentzian structure $(M, \stackrel{\circ}{\mathbf{g}}, \stackrel{\circ}{D})$. Those results are important for a proper understanding of this paper.

\subsection{The Bianchi Identities}

Given a general Riemann-Cartan structure $(M, \mathbf{g}, D)$ we have the following identities

$$
\begin{aligned}
& \mathbf{D} \mathcal{T}^{\alpha}=\mathcal{R}_{\beta}^{\alpha} \wedge \theta^{\beta}, \\
& \mathbf{D} \mathcal{R}_{\beta}^{\alpha}=0,
\end{aligned}
$$

known respectively as the first and second Bianchi identities (see e.g., [8, 23]). In the above equations, $\mathbf{D}$ is the exterior covariant derivative of indexed form fields [4, 23, whose precise definition is recalled in Appendix A. Now, the coordinate expressions of Eqs.(18) and (19) can easily be found and are respectively [8, 24] written as

$$
\begin{aligned}
\sum_{(\mu \alpha \beta)} R_{\mu}{ }^{\rho} \alpha \beta & =\sum_{(\mu \alpha \beta)}\left(D_{\mu} T_{\alpha \beta}^{\rho}-T_{\mu \beta}^{\kappa} T_{\kappa \alpha}^{\rho}\right), \\
\sum_{(\mu \nu \rho)} D_{\mu} R_{\beta \nu \rho}^{\alpha} & =\sum_{(\mu \nu \rho)} T_{\nu \mu}^{\kappa} R_{\beta \kappa \rho}^{\alpha},
\end{aligned}
$$

where $\sum_{(\mu \nu \rho)}$ denotes (as usual) the cyclic sum. For future use we observe that

$$
\mathcal{R}_{\beta}^{\alpha} \wedge \theta^{\beta}=\frac{1}{3 !}\left(R_{\mu}{ }^{\alpha}{ }_{\alpha \beta}+R_{\alpha}{ }^{\alpha}{ }_{\beta \mu}+R_{\beta}{ }^{\alpha}{ }_{\mu \alpha}\right) \theta^{\mu} \wedge \theta^{\alpha} \wedge \theta^{\beta}
$$

Remark 7 For a Lorentz structure $(M, \stackrel{\circ}{\mathrm{g}}, \stackrel{\circ}{D})$ the Bianchi identities reduce to

$$
\stackrel{\circ}{\mathcal{R}}_{\beta}^{\alpha} \wedge \theta^{\beta}=0, \quad \mathbf{D} \stackrel{\circ}{\mathcal{R}}_{\beta}^{\alpha}=0,
$$

or in components:

$$
\sum_{(\mu \alpha \beta)} R_{\mu}{ }^{\rho} \alpha \beta=0, \quad \sum_{(\mu \nu \rho)} D_{\mu} R_{\beta \nu \rho}^{\alpha}=0
$$

\section{Ricci and Einstein 1-form fields}

Given $R_{\mu \nu}$ and $G_{\mu \nu}$, respectively the components of the Ricci and Einstein tensors (in the general basis introduced above) we define the Ricci $\left(\mathcal{R}^{\mu} \in \sec \Lambda^{1} T^{*} M\right)$ and the Einstein $\left(\mathcal{G}^{\mu} \in \sec \Lambda^{1} T^{*} M\right)$ 1-form fields by

$$
\mathcal{R}^{\mu}:=R_{\nu}^{\mu} \theta^{\nu}, \quad \mathcal{G}^{\mu}:=G_{\nu}^{\mu} \theta^{\nu} .
$$

\footnotetext{
${ }^{7}$ In fact a gravitational field is defined by an equivalence class of pentuples, where $\left(M, g, D, \tau_{g}, \uparrow\right)$ and $\left(M^{\prime}, g^{\prime}, D^{\prime}, \tau_{g}^{\prime}, \uparrow^{\prime}\right)$ are said to be equivalent if there is a diffeomorphism $\mathrm{h}: M \rightarrow M^{\prime}$, such that $\mathrm{g}^{\prime}=\mathrm{h}^{*} \mathrm{~g}, D^{\prime}=\mathrm{h}^{*} D, \tau_{g}^{\prime}=\mathrm{h}^{*} \tau_{g}, \uparrow^{\prime}=\mathrm{h}^{*} \uparrow,\left(\right.$ where $\mathrm{h}^{*}$ here denotes the pullback mapping). For more details, see, e.g., 22] 23. With the above definition we exclude from our considerations models with closed timelike curves, which according to our view are pure science fiction.
} 
For future use we introduce also the energy-momentum 1-form fields $\mathbf{T}^{\mu} \in \sec \Lambda^{1} T^{*} M$ by

$$
\mathbf{T}^{\mu}:=T_{\nu}^{\mu} \theta^{\nu}
$$

Also

$$
\begin{aligned}
\underset{g}{\star \mathbf{T}^{\mu}} & =T_{\nu}^{\mu} \underset{g}{\mu} \theta^{\nu} \\
& =\frac{1}{3 !}\left(T_{\nu}^{\mu} \sqrt{|\operatorname{det} \mathbf{g}|} g^{\nu \kappa} \epsilon_{\kappa \iota \lambda \sigma}\right) \theta^{\iota} \wedge \theta^{\kappa} \wedge \theta^{\sigma} .
\end{aligned}
$$

Proposition 8 The dual of the Ricci and Einstein 1 -form fields, i.e., $\star \mathcal{R}^{\alpha} \in \sec \Lambda^{3} T^{*} M$ and $\star \mathcal{G}^{\alpha} \in \sec \Lambda^{3} T^{*} M$ can be written as:

$$
\begin{aligned}
& \underset{\mathrm{g}}{\star \mathcal{R}^{\alpha}}=-\underset{\mathrm{g}}{\star} \mathcal{R}_{\beta}^{\alpha} \wedge \theta^{\beta}=-\theta^{\beta} \wedge \underset{\mathrm{g}}{\star \mathcal{R}_{\beta}^{\alpha}}, \\
& \underset{\mathrm{g}}{\star \mathcal{G}^{\rho}}=-\frac{1}{2} \mathcal{R}_{\alpha \beta} \wedge \underset{\mathrm{g}}{\star}\left(\theta^{\alpha} \wedge \theta^{\beta} \wedge \theta^{\rho}\right) .
\end{aligned}
$$

where $\mathcal{R}_{\mu}^{\rho}=\frac{1}{2} R_{\mu}{ }^{\rho}{ }_{\alpha \beta} \theta^{\alpha} \wedge \theta^{\beta}$ and $\mathcal{R}_{\mu \rho}:=\frac{1}{2} R_{\mu \rho \alpha \beta} \theta^{\alpha} \wedge \theta^{\beta}$

Proof. Using some of the identities in Appendix E we can write immediately

$$
\begin{aligned}
\theta^{\rho} \underset{g}{\wedge} \mathcal{R}_{\mu \rho} & =-\underset{g}{\star}\left(\theta^{\rho} \underset{g}{\lrcorner} \mathcal{R}_{\mu \rho}\right) \\
& =-{ }_{g}^{\star} \frac{1}{2}\left[R_{\mu \rho \alpha \beta} \theta^{\rho} \underset{g}{\lrcorner}\left(\theta^{\alpha} \wedge \theta^{\beta}\right)\right]=-\underset{g}{\star}\left(R_{\mu \rho \alpha \beta} g^{\rho \alpha} \theta^{\beta}\right) \\
& =-{ }_{g}\left(R_{\mu \alpha \beta}^{\alpha} \theta^{\beta}\right)=-\star\left(R_{\mu \beta} \theta^{\beta}\right) \\
& =-\underset{g}{\star} \mathcal{R}_{\mu},
\end{aligned}
$$

and Eq.(26) is proved.

Now Eq.(27) is evinced. By taking some of the identities in Appendix E, we can immediately write:

$$
\begin{aligned}
& \left.\frac{1}{2} \mathcal{R}_{\alpha \beta} \wedge \underset{g}{\star}\left(\theta^{\alpha} \wedge \theta^{\beta} \wedge \theta^{\rho}\right)=-\frac{1}{2} \underset{\mathrm{g}}{\star}\left[\mathcal{R}_{\alpha \beta}\right\lrcorner \underset{g}{ }\left(\theta^{\alpha} \wedge \theta^{\beta} \wedge \theta^{\rho}\right)\right] \\
& =-\frac{1}{4} R_{\alpha \beta \iota \kappa} \underset{g}{\star}\left[\left(\theta^{\iota} \wedge \theta^{\kappa}\right) \underset{g}{\lrcorner}\left(\theta^{\alpha} \wedge \theta^{\beta} \wedge \theta^{\rho}\right)\right]=-\frac{1}{4} R_{\alpha \beta \iota \kappa} \underset{g}{\star}\left[\left(\theta_{g}^{\iota} \underset{g}{\lrcorner}\left(\theta^{\kappa}\right\lrcorner\left(\theta^{\alpha} \wedge \theta^{\beta} \wedge \theta^{\rho}\right)\right)\right] \\
& =-\underset{\mathrm{g}}{\star}\left(\mathcal{R}^{\rho}-\frac{1}{2} R \theta^{\rho}\right)
\end{aligned}
$$

and Eq.(27) is proved.

Remark 9 Recall that

$$
\begin{aligned}
\underset{\mathrm{g}}{\star \mathcal{R}_{\mu \rho}} & :=\frac{1}{2} R_{\mu \rho \alpha \beta}^{\star} \underset{\mathrm{g}}{ }\left(\theta^{\alpha} \wedge \theta^{\beta}\right) \\
& =\frac{1}{2} R_{\mu \rho \alpha \beta} \frac{1}{2} \sqrt{|\operatorname{det} \boldsymbol{g}|} g^{\alpha \kappa} g^{\beta \iota} \epsilon_{\kappa \iota \lambda \sigma} \theta^{\lambda} \wedge \theta^{\sigma}=\frac{1}{2}\left(\frac{1}{2} \sqrt{|\operatorname{det} \mathbf{g}|} \epsilon_{\kappa \iota \lambda \sigma} R_{\mu \rho}^{\kappa \iota}\right) \theta^{\lambda} \wedge \theta^{\sigma} \\
& =\frac{1}{2} \mathrm{R}_{\mu \rho \lambda \sigma}^{\star} \theta^{\lambda} \wedge \theta^{\sigma} \\
& =\frac{1}{2} R_{\mu \rho \lambda \sigma}^{\star} \sqrt{|\operatorname{det} \boldsymbol{g}|} \theta^{\lambda} \wedge \theta^{\sigma}
\end{aligned}
$$

with

$$
\mathrm{R}_{\mu \rho \lambda \sigma}^{\star}:=\frac{1}{2} \sqrt{|\operatorname{det} \boldsymbol{g}|} \epsilon_{\kappa \iota \lambda \sigma} R_{\alpha \beta}^{\kappa \iota}, \quad \text { and } \quad R_{\mu \rho \lambda \sigma}^{\star}:=\frac{1}{2} \epsilon_{\kappa \iota \lambda \sigma} R_{\alpha \beta}^{\kappa \iota} \text {, }
$$


and so it follows that

$$
\begin{aligned}
\star \mathcal{R}_{\mu \rho} \wedge \theta^{\rho} & =\frac{1}{2} \mathrm{R}_{\mu \rho \lambda \sigma}^{\star} \theta^{\lambda} \wedge \theta^{\sigma} \wedge \theta^{\rho}=\frac{1}{2} \mathrm{R}_{\mu \rho \lambda \sigma}^{\star} \theta^{\rho} \wedge \theta^{\lambda} \wedge \theta^{\sigma} \\
& =\frac{1}{2}\left(\frac{1}{3} \mathrm{R}_{\mu \rho \lambda \sigma}^{\star} \theta^{\rho} \wedge \theta^{\lambda} \wedge \theta^{\sigma}+\frac{1}{3} \mathrm{R}_{\mu \lambda \sigma \rho}^{\star} \theta^{\lambda} \wedge \theta^{\sigma} \wedge \theta^{\rho}+\frac{1}{3} \mathrm{R}_{\mu \sigma \rho \lambda}^{\star} \theta^{\sigma} \wedge \theta^{\rho} \wedge \theta^{\lambda}\right) \\
& =\frac{1}{3 !}\left(\mathrm{R}_{\mu \rho \lambda \sigma}^{\star}+\mathrm{R}_{\mu \lambda \sigma \rho}^{\star}+\mathrm{R}_{\mu \sigma \rho \lambda}^{\star}\right) \theta^{\rho} \wedge \theta^{\lambda} \wedge \theta^{\sigma} \\
& =\frac{1}{3 !}\left(R_{\mu \rho \lambda \sigma}^{\star}+R_{\mu \lambda \sigma \rho}^{\star}+R_{\mu \sigma \rho \lambda}^{\star}\right) \sqrt{|\operatorname{det} \boldsymbol{g}|} \theta^{\rho} \wedge \theta^{\lambda} \wedge \theta^{\sigma} .
\end{aligned}
$$

and taking into account Eq.(26) it reads:

$$
\underset{\mathrm{g}}{\star} \mathcal{R}_{\mu}=-\frac{1}{3 !}\left(R_{\mu \rho \lambda \sigma}^{\star}+R_{\mu \lambda \sigma \rho}^{\star}+R_{\mu \sigma \rho \lambda}^{\star}\right) \sqrt{|\operatorname{det} \boldsymbol{g}|} \theta^{\rho} \wedge \theta^{\lambda} \wedge \theta^{\sigma} .
$$

\section{The Dual of Einstein Equation in Riemann-Cartan Theory}

We now return to Eq.(23) which in components can read 8

$$
R_{\mu \nu}-\frac{1}{2} g_{\mu \nu} R=T_{\mu \nu}
$$

Multiplying this equation on both sides by $\theta^{\nu}$ and recalling the definitions of the Ricci, Einstein, and the energy-momentum 1-form fields given above we have

$$
\mathcal{G}_{\mu}=\mathbf{T}_{\mu}
$$

Taking the dual of this equation we get

$$
\underset{\mathrm{g}}{\star} \mathcal{G}_{\mu}=\underset{\mathrm{g}}{\star} \mathcal{R}_{\mu}-\frac{1}{2} R \underset{\mathrm{g}}{\star} \theta_{\mu}=\underset{\mathrm{g}}{\star \mathbf{T}_{\mu}}
$$

Taking Eq.(32) and Eq.(25) into account, then Eq.(35) can be expressed as

$$
\begin{aligned}
& -\frac{1}{3 !}\left(R_{\mu \rho \lambda \sigma}^{\star}+R_{\mu \lambda \sigma \rho}^{\star}+R_{\mu \sigma \rho \lambda}^{\star}+\frac{1}{2} R \delta_{\mu}^{\kappa} \epsilon_{\kappa \rho \lambda \sigma}\right) \sqrt{|\operatorname{det} \boldsymbol{g}|} \theta^{\rho} \wedge \theta^{\lambda} \wedge \theta^{\sigma} \\
& =\frac{1}{3 !}\left(T_{\mu \nu} g^{\nu \kappa} \epsilon_{\kappa \rho \lambda \sigma}\right) \sqrt{|\operatorname{det} \boldsymbol{g}|} \theta^{\rho} \wedge \theta^{\lambda} \wedge \theta^{\sigma},
\end{aligned}
$$

or equivalently

$$
\left(R_{\mu \rho \lambda \sigma}^{\star}+R_{\mu \lambda \sigma \rho}^{\star}+R_{\mu \sigma \rho \lambda}^{\star}+\frac{1}{2} R \epsilon_{\mu \rho \lambda \sigma}\right)=\epsilon_{\rho \lambda \sigma \kappa} T_{\mu}^{\kappa} .
$$

\subsection{The Field and Structure Equations}

We now summarize the field and Bianchi identities for a Riemann-Cartan theory where an Einstein-like equation holds. Those equations can be written conveniently in intrinsic and component forms respectively as:

$$
\begin{aligned}
& \underset{\mathrm{g}}{\star \mathcal{G}_{\mu}}=\underset{\mathrm{g}}{\star \mathcal{R}_{\mu}}-\frac{1}{2} R \underset{\mathrm{g}}{\star} \theta_{\mu}=\underset{\mathrm{g}}{\star} \mathbf{T}_{\mu} \\
& \mathbf{D} \mathcal{T}^{\alpha}=\mathcal{R}_{\beta}^{\alpha} \wedge \theta^{\beta}, \\
& \mathbf{D} \mathcal{R}_{\beta}^{\alpha}=0 \text {, }
\end{aligned}
$$

\footnotetext{
${ }^{8}$ Take notice that in Eq. (33) $R_{\mu \nu}$ and $T_{\mu \nu}$ are not symmetric.
} 


$$
\begin{aligned}
\left(R_{\mu \rho \lambda \sigma}^{\star}+R_{\mu \lambda \sigma \rho}^{\star}+R_{\mu \sigma \rho \lambda}^{\star}+\frac{1}{2} R \epsilon_{\mu \rho \lambda \sigma}\right) & =\epsilon_{\rho \lambda \sigma \kappa} T_{\mu}^{\kappa} \Longleftrightarrow G_{\mu \nu}=T_{\mu \nu}, \\
\sum_{(\mu \alpha \beta)} R_{\mu \rho \alpha \beta} & =\sum_{(\mu \alpha \beta)}\left(D_{\mu} T_{\rho \alpha \beta}-T_{\mu \beta}^{\kappa} T_{\rho \kappa \alpha}\right), \\
\sum_{(\mu \nu \rho)} D_{\mu} R_{\beta \alpha \nu \rho} & =\sum_{(\mu \nu \rho)} T_{\nu \mu}^{\kappa} R_{\beta \alpha \kappa \rho} .
\end{aligned}
$$

In a GRT model it follows that

$$
\begin{aligned}
\left(\stackrel{\circ}{R}_{\mu \rho \lambda \sigma}^{\star}+\stackrel{\circ}{R}_{\mu \lambda \sigma \rho}^{\star}+\stackrel{\circ}{R}_{\mu \sigma \rho \lambda}^{\star}+\frac{1}{2} \stackrel{\circ}{R} \epsilon_{\mu \rho \lambda \sigma}\right) & =\epsilon_{\rho \lambda \sigma \kappa} \stackrel{\circ}{T}_{\mu}^{\kappa} \Leftrightarrow G_{\mu \nu}=T_{\mu \nu}, \\
\sum_{(\mu \alpha \beta)} \stackrel{\circ}{R}_{\mu \rho \alpha \beta} & =0, \quad \sum_{(\mu \nu \rho)} \stackrel{\circ}{D}_{\mu} \stackrel{\circ}{R}_{\beta \alpha \nu \rho}=0 .
\end{aligned}
$$

Remark 10 Before proceeding we want to emphasize that the Eq.(18) and Eq.(19) (the Bianchi identities) do not imply in general in the validity of the analogous equations for the duals of the torsion and curvature 2-forms, i.e., in genera包

$$
\begin{aligned}
& \mathbf{D} \underset{\mathrm{g}}{\star \mathcal{T}^{\alpha}} \neq \underset{\mathrm{g}}{\star \mathcal{R}_{\beta}^{\alpha} \wedge \theta^{\beta},} \\
& \mathbf{D} \underset{\mathrm{g}}{\mathrm{g}} \mathcal{R}_{\beta}^{\alpha} \neq 0 .
\end{aligned}
$$

\section{$5 \quad$ Are $\star \mathcal{T}^{\alpha}$ and $\star \mathcal{R}_{\beta}^{\alpha}$ the Torsion and Curvature 2-Forms of any Con- nection?}

Despite the fact aforementioned in the last Remark, we may pose the question: can $\underset{\mathrm{g}}{\star} \mathcal{T}^{\alpha}$ and $\underset{\mathrm{g}}{\star \mathcal{R}_{\beta}}$ be the torsion and curvature 2-forms of a $g$-metric compatible connection, say $D^{\prime}$, which defines on $M$ the Riemann-Cartan structure $\left(M, \mathbf{g}, D^{\prime}\right)$ where also an Einstein like equation is valid? If the answer is positive, the following set of equations must hold:

$$
\begin{aligned}
{ }_{\mathrm{g}} \mathcal{G}_{\mu}^{\prime} & =\underset{\mathrm{g}}{\star \mathcal{R}_{\mu}^{\prime}}-\frac{1}{2} R_{\mathrm{g}}^{\star{ }_{\mathrm{g}}} \theta_{\mu}=\underset{\mathrm{g}}{\star \mathbf{T}_{\mu}^{\prime}} \\
\mathbf{D}^{\prime} \mathcal{T}^{\prime \alpha} & =\mathcal{R}_{\beta}^{\prime \alpha} \wedge \theta^{\beta} \\
\mathbf{D}^{\prime} \mathcal{R}_{\beta}^{\prime \alpha} & =0
\end{aligned}
$$

and since by hypothesis $\underset{\mathrm{g}}{\star \mathcal{T}^{\alpha}}=\mathcal{T}^{\prime \alpha}$ and $\underset{\mathrm{g}}{\star \mathcal{R}_{\beta}} \underset{\beta}{\alpha}=\mathcal{R}_{\beta}^{\prime \alpha}$, calling $\mathcal{G}_{\mu}^{*}=\mathcal{G}_{\mu}^{\prime}, \mathcal{R}_{\mu}^{*}=\mathcal{R}_{\mu}^{\prime}, R^{*}=R^{\prime}=g^{\mu \beta} R_{\mu \rho \beta}^{*^{\rho}}$ it must be

$$
\begin{aligned}
& \underset{\mathrm{g}}{\star \mathcal{G}_{\mu}^{*}}=\underset{\mathrm{g}}{\star \mathcal{R}_{\mu}^{*}}-\frac{1}{2} R_{\mathrm{g}}^{*} \underset{\mathrm{g}}{\star \theta_{\mu}}=\underset{\mathrm{g}}{\star \mathbf{T}_{\mu}^{\prime}} \\
& \mathbf{D}^{\prime} \underset{\mathrm{g}}{\star \mathcal{T}^{\alpha}}=\underset{\mathrm{g}}{\star \mathcal{R}_{\beta}} \mathcal{A}_{\beta} \wedge \theta^{\beta}, \\
& \mathbf{D}^{\prime} \star \mathcal{R}_{\beta}^{\alpha}=0 \text {. }
\end{aligned}
$$

or in component form (and obvious notation)

$$
\begin{aligned}
\left(R_{\mu \rho \lambda \sigma}+R_{\mu \lambda \sigma \rho}+R_{\mu \sigma \rho \lambda}+\frac{1}{2} R^{*} \epsilon_{\mu \rho \lambda \sigma}\right) & =\epsilon_{\rho \lambda \sigma \kappa} T_{\mu}^{\prime \kappa} \Longleftrightarrow G_{\mu \nu}^{\prime}=T_{\mu \nu}^{\prime}, \\
\sum_{(\mu \alpha \beta)} R_{\mu \rho \alpha \beta}^{*} & =\sum_{(\mu \alpha \beta)}\left(D_{\mu}^{\prime} T_{\rho \alpha \beta}^{*}-T_{\mu \beta}^{* \kappa} T_{\rho \kappa \alpha}^{*}\right), \\
\sum_{(\mu \nu \rho)} D_{\mu}^{\prime} R_{\beta \alpha \nu \rho}^{*} & =\sum_{(\mu \nu \rho)} T_{\nu \mu}^{* \kappa} R_{\beta \alpha \kappa \rho}^{*}
\end{aligned}
$$

\footnotetext{
${ }^{9}$ In particular, a correct expression for $\mathbf{D} \underset{\mathrm{g}}{\star \mathcal{T}^{\alpha}}$ has been found in 24.
} 
Consequently, among the possible constraints in order to have a positive answer concerning the question in the head of the Section, the following two non trivial constraints are derived:

(a) Using Eq.(40) and Eq.(49), it follows that

$$
\epsilon_{\rho \lambda \sigma \kappa} T_{\mu}^{\kappa}-\frac{1}{2} R \epsilon_{\mu \rho \lambda \sigma}=\sum_{(\mu \alpha \beta)}\left(D_{\mu}^{\prime} T_{\rho \alpha \beta}^{*}-T_{\mu \beta}^{*} T_{\rho \kappa \alpha}^{*}\right) .
$$

(b) Using Eq.(48) and Eq.(41) we must have

$$
\epsilon_{\rho \lambda \sigma \kappa} T_{\mu}^{\prime \kappa}-\frac{1}{2} R^{*} \epsilon_{\mu \rho \lambda \sigma}=\sum_{(\mu \alpha \beta)}\left(D_{\mu} T_{\rho \alpha \beta}-T_{\mu \beta}^{\kappa} T_{\rho \kappa \alpha}\right) .
$$

Let us analyze what those constraints imply if we start with $(M, \mathbf{g}, \stackrel{\circ}{D})$, a Lorentzian structure (part of a Lorentzian spacetime structure) representing a gravitational field in GRT. In this case the second member of Eq. (52) must equal zero, and taking into account that $\stackrel{\circ}{R}=\stackrel{\circ}{T}^{\prime}:=\stackrel{\circ}{\kappa}_{\kappa}^{\kappa}$ and $\stackrel{\circ}{R}^{*}=-\dot{T}^{\prime}:=\dot{T}_{\kappa}^{\prime \kappa}$ we get that the structure $\left(M, \mathbf{g}, \stackrel{\circ}{D}^{\prime}\right)$ must also be torsion free and the following constraints must hold:

$$
\begin{array}{rlrl}
\epsilon_{\rho \lambda \sigma \kappa} \stackrel{\circ}{T}_{\mu}^{\kappa} & =-\frac{1}{2} \stackrel{\circ}{T} \epsilon_{\mu \rho \lambda \sigma}, & \epsilon_{\rho \lambda \sigma \kappa} \stackrel{\circ}{T}_{\mu}^{\prime \kappa}=-\frac{1}{2} \stackrel{\circ}{T}^{\prime} \epsilon_{\mu \rho \lambda \sigma}, \\
\sum_{(\mu \nu \rho)} \stackrel{\circ}{D}_{\mu}^{\prime} R_{\beta \alpha \nu \rho}^{*} & =\sum_{(\mu \nu \rho)} \stackrel{\circ}{D}_{\mu} R_{\beta \alpha \nu \rho} .
\end{array}
$$

\section{A Particular Case}

Suppose we have as postulated 10 in (1] a Riemann-Cartan structure where Eq.(40), Eq.(41), and Eq.(42) read:

$$
\begin{aligned}
\left(R_{\mu \rho \lambda \sigma}^{\star}+R_{\mu \lambda \sigma \rho}^{\star}+R_{\mu \sigma \rho \lambda}^{\star}\right) & =\epsilon_{\rho \lambda \sigma \kappa} T_{\mu}^{\kappa} \Longleftrightarrow G_{\mu \nu}=T_{\mu \nu}, \\
\sum_{(\mu \alpha \beta)} R_{\mu \rho \alpha \beta} & =\epsilon_{\rho \alpha \beta \kappa} \Theta_{\mu}^{\kappa}, \\
\sum_{(\mu \nu \rho)} D_{\mu} R_{\beta \alpha \nu \rho} & =0 .
\end{aligned}
$$

It is obvious that we must then have:

$$
R=0, \quad \epsilon_{\rho \alpha \beta \kappa} \Theta_{\mu}^{\kappa}=\sum_{(\mu \alpha \beta)}\left(D_{\mu} T_{\rho \alpha \beta}-T_{\mu \beta}^{\kappa} T_{\rho \kappa \alpha}\right), \quad \sum_{(\mu \nu \rho)} T_{\nu \mu}^{\kappa} R_{\beta \alpha \kappa \rho}=0,
$$

and comparing Eq.(52) with Eq.(157) we get

$$
\epsilon_{\rho \lambda \sigma \kappa} T_{\mu}^{\prime \kappa}+\frac{1}{2} T_{\kappa}^{\prime \kappa} \epsilon_{\mu \rho \lambda \sigma}=\epsilon_{\rho \alpha \beta \kappa} \Theta_{\mu}^{\kappa} .
$$

So a Riemann-Cartan structure satisfying Eq.(54), Eq.(55) and Eq.(56) is possible only for matter distributions with $T=T_{\kappa}^{\kappa}=0$ and which obey very stringent constraints.

Also, [1] choose as "dual equations" the following set:

$$
\begin{aligned}
\left(R_{\mu \rho \lambda \sigma}+R_{\mu \lambda \sigma \rho}+R_{\mu \sigma \rho \lambda}+\frac{1}{2} R^{*} \epsilon_{\mu \rho \lambda \sigma}\right) & =\epsilon_{\rho \lambda \sigma \kappa} \Theta_{\mu}^{\kappa} \Longleftrightarrow G_{\mu \nu}^{*}=\Theta_{\mu \nu}, \\
\sum_{(\mu \alpha \beta)} R_{\mu \rho \alpha \beta}^{*} & =\epsilon_{\rho \alpha \beta \kappa} T_{\mu}^{\kappa}, \\
\sum_{(\mu \nu \rho)} D_{\mu}^{\prime} R_{\beta \alpha \nu \rho}^{*} & =0
\end{aligned}
$$

\footnotetext{
${ }^{10}$ It is obvious from our previous considerations that the equation $\left(R_{\mu \rho \lambda \sigma}^{\star}+R_{\mu \lambda \sigma \rho}^{\star}+R_{\mu \sigma \rho \lambda}^{\star}\right)=\epsilon_{\rho \lambda \sigma \kappa} T_{\mu}{ }^{\kappa}$ presented in [1] as an identity is in general wrong and invalidates most of the conclusions of that paper. Take also notice that in 1 it is defined a Hodge dual with respect to the first pair of indices. However, since they start from a Lorentzian structure (where torsion is null) we have the validity of Eq.99) and so it does not matter in deriving Eq. (40) taking the dual with respect to the first or second pair of indices.
} 
which, of course must imply

$$
\begin{aligned}
R^{*} & =0, \\
\epsilon_{\rho \alpha \beta \kappa} T_{\mu}^{\kappa} & =\sum_{(\mu \alpha \beta)}\left(D_{\mu}^{\prime} T_{\rho \alpha \beta}^{*}-T_{\mu \beta}^{*}{ }^{*} T_{\rho \kappa \alpha}^{*}\right), \\
\sum_{(\mu \nu \rho)} T_{\nu \mu}^{* \kappa} R_{\beta \alpha \kappa \rho}^{*} & =0 .
\end{aligned}
$$

Comparing Eq.(64) to Eq.(51) implies again that $R=0$. So we end with the following constraints, necessary for the validity of the equations proposed in [1]:

$$
\begin{aligned}
& T_{\mu}^{\prime \kappa}=\Theta_{\mu}^{\kappa}, \quad T=T_{\kappa}^{\kappa}=0, \quad \Theta=\Theta_{\kappa}^{\kappa}=0, \\
& \epsilon_{\rho \alpha \beta \kappa} \Theta_{\mu}^{\kappa}=\sum_{(\mu \alpha \beta)}\left(D_{\mu} T_{\rho \alpha \beta}-T_{\mu \beta}^{\kappa} T_{\rho \kappa \alpha}\right), \quad \epsilon_{\rho \alpha \beta \kappa} T_{\mu}^{\kappa}=\sum_{(\mu \alpha \beta)}\left(D_{\mu}^{\prime} T_{\rho \alpha \beta}^{*}-T_{\mu \beta}^{* \kappa} T_{\rho \kappa \alpha}^{*}\right), \\
& \sum_{(\mu \nu \rho)} T_{\nu \mu}^{\kappa} R_{\beta \alpha \kappa \rho}=0, \sum_{(\mu \nu \rho)} T_{\nu \mu}^{* \kappa} R_{\beta \alpha \kappa \rho}^{*}=0 .
\end{aligned}
$$

Such constraints are clearly violated by the examples in [1].

\section{Is there a metric $g^{\prime}$ and a metric connection $D^{\prime}$ such that $\underset{g^{\prime}}{\star} \mathcal{T}^{\alpha}$ and

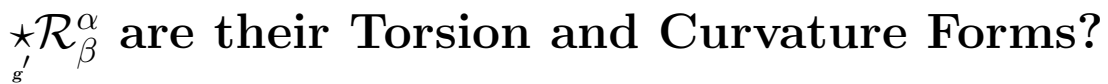

Now, we can also put the question: in which conditions may we conceive that $\underset{\mathrm{g}}{\star \mathcal{T}^{\alpha}}$ and $\underset{g^{\prime}}{\star} \mathcal{R}_{\beta}^{\alpha}$ are the torsion and curvature 2 -forms of a $g^{\prime}$-metric compatible connection, say $D^{\prime}$ which defines on $M$ the Riemann-Cartan structure $\left(M, \mathbf{g}^{\prime}, D^{\prime}\right)$ where an Einstein like equation holds, i.e., (with obvious notation) the validity of the following set of equations $\left(R^{\prime}=g^{\prime \mu \beta} R_{\mu \rho \beta}^{\prime \rho}\right)$ :

$$
\begin{aligned}
& \underset{g^{\prime}}{\star} \mathcal{G}_{\mu}^{\prime}=\underset{g^{\prime}}{\star} \mathcal{R}_{\mu}^{\prime}-\frac{1}{2} R_{\underset{g^{\prime}}{\star}}^{\star} \theta_{\mu}=\underset{g^{\prime}}{\star} \mathbf{T}_{\mu}^{\prime} \\
& \mathbf{D}^{\prime} \mathcal{T}^{\prime \alpha}=\mathcal{R}_{\beta}^{\prime \alpha} \wedge \theta^{\beta}, \\
& \mathbf{D}^{\prime} \mathcal{R}_{\beta}^{\prime \alpha}=0 .
\end{aligned}
$$

Since by hypothesis we must have $\underset{\mathrm{g}}{\star} \mathcal{T}^{\alpha}=\mathcal{T}^{\prime \alpha}$ and $\underset{\mathrm{g}}{\star \mathcal{R}_{\beta}} \boldsymbol{\alpha}=\mathcal{R}_{\beta}^{\prime \alpha}$, calling $R^{*}=g^{\prime \mu \beta} R_{\mu \rho \beta}^{*^{\rho}}$ the set of Eqs.(66) must be equal to:

$$
\begin{aligned}
& \underset{g^{\prime}}{\star} \mathcal{G}_{\mu}^{*}=\underset{g^{\prime}}{\star} \mathcal{R}_{\mu}^{*}-\frac{1}{2} R_{g_{g^{\prime}}^{*}}^{\star} \theta_{\mu}=\underset{g^{\prime}}{\star} \mathbf{T}_{\mu}^{\prime} \\
& \mathbf{D}^{\prime} \star_{\mathrm{g}} \mathcal{T}^{\prime \alpha}={ }_{\mathrm{g}} \mathcal{R}_{\beta}^{\alpha} \wedge \theta^{\beta}, \\
& \mathbf{D}_{\mathrm{g}}^{\prime \star \mathcal{R}_{\beta}^{\prime \alpha}}=0 \text {. }
\end{aligned}
$$

which are similar but not identical to the set given by Eq.(47). Due to their complexity we shall not inspect the nature of those equations solutions, a problem postponed for another publication.

Remark 11 The constraints concerned in this case are more involved than in the previous case, but we want to emphasize here that if we start with $(M, \mathbf{g}, \stackrel{\circ}{D})$, a Lorentzian structure (part of a Lorentzian spacetime structure) representing a gravitational field in GRT, the structure $\left(M, \mathbf{g}^{\prime}, \stackrel{\circ}{D}^{\prime}\right)$ will be also torsion free. Here we recalled that [9] investigated long ago a similar problem (but only in the linear approximation) and found a positive answer for the question at the head of this Section. 


\section{Conclusions}

In this paper we present the correct constraints that must be satisfied by any theory (in a 4-dimensional manifold) that intends to provide a dual presentation of the gravitational field equations for a general Riemann-Cartan theory. We compare our results with some ones proposed by authors quoted in the introduction and present some constructive criticisms. We hope that since the subject of duality becomes each day more important in non, e.g., abelian gauge theories, gravity and $M$-theory that our results shall become appreciated.

\section{A Exterior Covariant Derivative D}

Sometimes Eqs.(13) are written by some authors as:

$$
\mathbf{D} \theta^{\rho}=\mathcal{T}^{\rho}, \quad \text { "D } \omega_{\mu}^{\rho}=\mathcal{R}_{\mu}^{\rho} .
$$

and $\mathbf{D}: \sec \Lambda T^{*} M \rightarrow \sec \Lambda T^{*} M$ is said to be the exterior covariant derivative related to the connection $D$. The second of Eqs. (68) has been printed with quotation marks due to the fact that it is not a correct equation. Indeed, a legitimate exterior covariant derivative operator 11 is a concept that can be defined for $(p+q)$-indexed $r$-form fields 122 as follows. Suppose that $X \in \sec T_{p}^{r+q} M$ and let $X_{\nu_{1} \ldots \nu_{q}}^{\mu_{1} \ldots \mu_{p}} \in \sec \Lambda^{r} T^{*} M$, such that for $v_{i} \in \sec T M, i=0,1,2, \ldots, r$, then $X_{\nu_{1} \ldots \nu_{q}}^{\mu_{1} \ldots \mu_{p}}\left(v_{1}, \ldots, v_{r}\right)=X\left(v_{1}, \ldots, v_{r}, e_{\nu_{1}}, \ldots, e_{\nu_{q}}, \theta^{\mu_{1}}, \ldots, \theta^{\mu_{p}}\right)$. The exterior covariant differential $\mathbf{D}$ of $X_{\nu_{1} \ldots \nu_{q}}^{\mu_{1} \ldots \mu_{p}}$ on a manifold with a general connection $D$ is the mapping

$$
\mathbf{D}: \sec \Lambda^{r} T^{*} M \rightarrow \sec \Lambda^{r+1} T^{*} M, \quad 0 \leq r \leq 4,
$$

such that 13

$$
\begin{aligned}
& (r+1) \mathbf{D} X_{\nu_{1} \ldots \nu_{q}}^{\mu_{1} \ldots \mu_{p}}\left(v_{0}, v_{1}, \ldots, v_{r}\right) \\
& =\sum_{\nu=0}^{r}(-1)^{\nu} D_{\mathbf{e}_{\nu}} X\left(v_{0}, v_{1}, \ldots, \check{v}_{\nu}, \ldots, v_{r}, \mathbf{e}_{\nu_{1}}, \ldots, \boldsymbol{e}_{\nu_{q}}, \theta^{\mu_{1}}, \ldots, \theta^{\mu_{p}}\right) \\
& \quad-\sum_{0 \leq \lambda, \varsigma \leq r}(-1)^{\nu+\varsigma} X\left(\mathbf{T}\left(v_{\lambda}, v_{\varsigma}\right), v_{0}, v_{1}, \ldots, \check{v}_{\lambda}, \ldots, \check{v}_{\varsigma}, \ldots, v_{r}, \mathbf{e}_{\nu_{1}}, \ldots, \mathbf{e}_{\nu_{q}}, \theta^{\mu_{1}}, \ldots, \theta^{\mu_{p}}\right) .
\end{aligned}
$$

Then, we may verify that

$$
\mathbf{D} X_{\nu_{1} \ldots \nu_{q}}^{\mu_{1} \ldots \mu_{p}}=d X_{\nu_{1} \ldots \nu_{q}}^{\mu_{1} \ldots \mu_{p}}+\omega_{\mu_{s}}^{\mu_{1}} \wedge X_{\nu_{1} \ldots \nu_{q}}^{\mu_{s} \ldots \mu_{p}}+\cdots+\omega_{\mu_{s}}^{\mu_{1}} \wedge X_{\nu_{1} \ldots \nu_{q}}^{\mu_{1} \ldots \mu_{p}}-\omega_{\nu_{1}}^{\nu_{s}} \wedge X_{\nu_{s} \ldots \nu_{q}}^{\mu_{1} \ldots \mu_{p}}-\cdots-\omega_{\mu_{s}}^{\mu_{1}} \wedge X_{\nu_{1} \ldots \nu_{s}}^{\mu_{1} \ldots \mu_{p}} .
$$

Remark 12 Note that if Eq.(71) is applied on any one of the connection 1-forms $\omega_{\nu}^{\mu}$ we would get $\mathbf{D} \omega_{\nu}^{\mu}=$ $d \omega_{\nu}^{\mu}+\omega_{\alpha}^{\mu} \wedge \omega_{\nu}^{\alpha}-\omega_{\nu}^{\alpha} \wedge \omega_{\alpha}^{\mu}$. So, we see that the symbol $\mathbf{D} \omega_{\nu}^{\mu}$ in Eq.(68), supposedly defining the curvature 2-forms, is simply wrong, despite this being an equation printed in many Physics textbooks and many professional articles.

\section{A.1 Properties of D}

The exterior covariant derivative $\mathbf{D}$ satisfy the following properties:

(a) For any $X^{J} \in \sec \Lambda^{r} T^{*} M$ and $Y^{K} \in \sec \Lambda^{s} T^{*} M$ are sets of indexed form: 14, then

$$
\mathbf{D}\left(X^{J} \wedge Y^{K}\right)=\mathbf{D} X^{J} \wedge Y^{K}+(-1)^{r s} X^{J} \wedge \mathbf{D} Y^{K} .
$$

(b) For any $X^{\mu_{1} \ldots \mu_{p}} \in \sec \Lambda^{r} T^{*} M$ then

$$
\mathbf{D D} X^{\mu_{1} \ldots \mu_{p}}=d X^{\mu_{1} \ldots \mu_{p}}+\mathcal{R}_{\mu_{s}}^{\mu_{1}} \wedge X^{\mu_{s} \ldots \mu_{p}}+\cdots+\mathcal{R}_{\mu_{s}}^{\mu_{p}} \wedge X^{\mu_{1} \ldots \mu_{s}} .
$$

(c) For any metric-compatible connection $D$ if $g=g_{\mu \nu} \theta^{\mu} \otimes \theta^{\nu}$ then, $\mathbf{D} g_{\mu \nu}=0$.

\footnotetext{
${ }^{11}$ Sometimes also called exterior covariant differential.

${ }^{12}$ Which is not the case of the connection 1-forms $\omega_{\beta}^{\alpha}$, despite the name. More precisely, the $\omega_{\beta}^{\alpha}$ are not true indexed forms, i.e., there does not exist a tensor field $\omega$ such that $\omega\left(e_{i}, e_{\beta}, \vartheta^{\alpha}\right)=\omega_{\beta}^{\alpha}\left(e_{i}\right)$.

${ }^{13}$ As usual the inverted hat over a symbol (in Eq. (70) ) means that the corresponding symbol is missing in the expression.

${ }^{14}$ Multi indices are here represented by $J$ and $K$.
} 


\section{B Relation Between the Riemann Curvature Tensors of the Levi- Civita Connection of $\stackrel{g}{g}$ and a g-compatible Riemann-Cartan Con- nection}

Let $(M, \stackrel{g}{g}, \stackrel{\circ}{D})$ and $(M, \mathbf{g}, D)$ be respectively a Lorentzian and a Riemann-Cartan structuret5 on the same manifold $M$ such that

$$
\stackrel{\circ}{\mathrm{g}}=0, \quad D \boldsymbol{g}=0,
$$

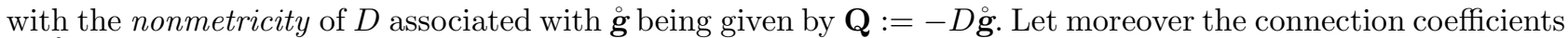
of $D^{\circ}$ and $D$ in the arbitrary bases dual bases $\left\{\mathbf{e}_{\alpha}\right\}$ and $\left\{\theta^{\rho}\right\}$ for $T U \subset T M$ and $T^{*} U \subset T^{*} M$ be:

$$
\stackrel{\circ}{D}_{\partial_{\alpha}} \theta^{\rho}=-\stackrel{\circ}{\Gamma}_{\alpha \beta}^{\rho} \theta^{\beta}, \quad D_{\partial_{\alpha}} \theta^{\rho}=-L_{\alpha \beta}^{\rho} \theta^{\beta},
$$

and $Q_{\alpha \beta \sigma}=-D_{\alpha} \stackrel{\circ}{g}_{\beta \sigma}$. Define the components of the strain tensor of the connection $D$ (associated with $\stackrel{\circ}{D}$ ) by

$$
S_{\alpha \beta}^{\rho}=\left(L_{\alpha \beta}^{\rho}+L_{\alpha \beta}^{\rho}\right)-\left(\stackrel{\circ}{\Gamma}_{\alpha \beta}^{\rho}+\stackrel{\circ}{\Gamma}_{\alpha \beta}^{\rho}\right)
$$

It is trivially established that

$$
L_{\alpha \beta}^{\rho}=\stackrel{\circ}{\Gamma}_{\alpha \beta}^{\rho}+\frac{1}{2} T_{\alpha \beta}^{\rho}+\frac{1}{2} S_{\alpha \beta}^{\rho} .
$$

where $\stackrel{\circ}{\Gamma}_{\alpha \beta}^{\rho}$ are the components of the Levi-Civita connection of $\boldsymbol{g}$ and $T_{\alpha \beta}^{\rho}$ are the components of the torsion tensor of $D^{16}$.

Eq.(77) can be used to relate the covariant derivatives with respect to the connections $D$ and $D$ of any tensor field on the manifold. In particular, recalling that $\stackrel{\circ}{D}_{\alpha} \stackrel{\circ}{g}_{\beta \sigma}=\mathbf{e}_{\alpha}\left(\stackrel{\circ}{g}_{\beta \sigma}\right)-\stackrel{\circ}{g}_{\mu \sigma} \stackrel{\circ}{\Gamma}_{\alpha \beta}^{\mu}-\stackrel{\circ}{g}_{\beta \mu} \stackrel{\circ}{\Gamma}_{\alpha \sigma}^{\mu}=0$, we get the expression of the nonmetricity tensor of $D$ in terms of the torsion and the strain, namely,

$$
Q_{\alpha \beta \sigma}=\frac{1}{2}\left(\stackrel{\circ}{g}_{\mu \sigma} T_{\alpha \beta}^{\mu}+\stackrel{\circ}{g}_{\beta \mu} T_{\alpha \sigma}^{\mu}\right)+\frac{1}{2}\left(\stackrel{\circ}{g}_{\mu \sigma} S_{\alpha \beta}^{\mu}+\stackrel{\circ}{g}_{\beta \mu} S_{\alpha \sigma}^{\mu}\right) .
$$

Eq.(78) can be inverted to yield the expression of the strain in terms of the torsion and the nonmetricity. We get:

$$
S_{\alpha \beta}^{\rho}=\stackrel{\circ}{g}^{\rho \sigma}\left(Q_{\alpha \beta \sigma}+Q_{\beta \sigma \alpha}-Q_{\sigma \alpha \beta}\right)-\stackrel{\circ}{g}^{\rho \sigma}\left(\stackrel{\circ}{g}_{\beta \mu} T_{\alpha \sigma}^{\mu}+\stackrel{\circ}{g}_{\alpha \mu} T_{\beta \sigma}^{\mu}\right) .
$$

From Eq.(78) and Eq.(79) it is clear that nonmetricity and strain can be used interchangeably in the description of the geometry of a Riemann-Cartan-Weyl space. In particular, we have the relation:

$$
Q_{\alpha \beta \sigma}+Q_{\sigma \alpha \beta}+Q_{\beta \sigma \alpha}=S_{\alpha \beta \sigma}+S_{\sigma \alpha \beta}+S_{\beta \sigma \alpha}, \quad \text { where } S_{\alpha \beta \sigma}=\stackrel{\circ}{g} \rho \sigma_{\alpha \beta}^{\rho} .
$$

In order to simplify our next equations, let us introduce the notation:

$$
K_{\alpha \beta}^{\rho}=L_{\alpha \beta}^{\rho}-\stackrel{\circ}{\Gamma}_{\alpha \beta}^{\rho}=\frac{1}{2}\left(T_{\alpha \beta}^{\rho}+S_{\alpha \beta}^{\rho}\right) .
$$

From Eq.79 it follows that:

$$
K_{\alpha \beta}^{\rho}=-\frac{1}{2} \stackrel{\circ}{g}^{\rho \sigma}\left(D_{\alpha} \stackrel{\circ}{g}_{\beta \sigma}+D_{\beta} \stackrel{\circ}{g}_{\sigma \alpha}-D_{\sigma} \stackrel{\circ}{g}_{\alpha \beta}\right)-\frac{1}{2} \stackrel{\circ}{g}^{\rho \sigma}\left(\stackrel{\circ}{g}_{\mu \alpha} T_{\sigma \beta}^{\mu}+\stackrel{\circ}{g}_{\mu \beta} T_{\sigma \alpha}^{\mu}-\stackrel{\circ}{g}_{\mu \sigma} T_{\alpha \beta}^{\mu}\right) .
$$

Note also that for $D \stackrel{g}{g}=0, K_{\alpha \beta}^{\rho}$ is the so-called contorsion tensor.

Returning to Eq.(77), we obtain now the relation between the curvature tensor $R_{\mu}{ }^{\rho}{ }_{\alpha \beta}$ associated with the connection $D$ and the Riemann curvature tensor $\stackrel{\circ}{R}_{\mu}{ }^{\rho} \alpha \beta$ of the Levi-Civita connection $D$ associated with the metric $g$. We get, by a straightforward calculation:

$$
R_{\mu}{ }^{\rho} \alpha \beta=\stackrel{\circ}{R}_{\mu}{ }^{\rho} \alpha \beta+J_{\mu}{ }^{\rho}[\alpha \beta],
$$

\footnotetext{
${ }^{15}$ Note that $(M, \mathbf{g}, D)$ and $(M, \stackrel{\circ}{g}, \stackrel{\circ}{D})$ are in general Riemann-Cartan-Weyl structures. More general formulas relating two arbitrary general connections may be found, e.g., in 23 .

${ }^{16}$ More details may be found, e.g., in 23 .
} 
where:

$$
J_{\mu}{ }^{\rho} \alpha \beta=\stackrel{\circ}{D}_{\alpha} K_{\beta \mu}^{\rho}-K_{\beta \sigma}^{\rho} K_{\alpha \mu}^{\sigma}=D_{\alpha} K_{\beta \mu}^{\rho}-K_{\alpha \sigma}^{\rho} K_{\beta \mu}^{\sigma}+K_{\alpha \beta}^{\sigma} K_{\sigma \mu}^{\rho} .
$$

Multiplying both sides of Eq.(83) by $\frac{1}{2} \theta^{\alpha} \wedge \theta^{\beta}$ we get:

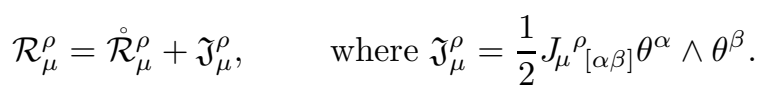

From Eq. (83) we also get the relation between the Ricci tensors of the connections $D$ and $\stackrel{\circ}{D}$. The Ricci tensor is defined by

$$
\text { Ricci }=R_{\mu \alpha} d x^{\mu} \otimes d x^{\nu}, \quad \text { where } \quad R_{\mu \alpha}:=R_{\mu}{ }^{\rho} \alpha \rho
$$

Then, we have

$$
R_{\mu \alpha}=\stackrel{\circ}{R}_{\mu \alpha}+J_{\mu \alpha}
$$

with

$$
\begin{aligned}
J_{\mu \alpha} & =\stackrel{\circ}{D}_{\alpha} K_{\rho \mu}^{\rho}-\stackrel{\circ}{D}_{\rho} K_{\alpha \mu}^{\rho}+K_{\alpha \sigma}^{\rho} K_{\rho \mu}^{\sigma}-K_{\rho \sigma}^{\rho} K_{\alpha \mu}^{\sigma} \\
& =D_{\alpha} K_{\rho \mu}^{\rho}-D_{\rho} K_{\alpha \mu}^{\rho}-K_{\sigma \alpha}^{\rho} K_{\rho \mu}^{\sigma}+K_{\rho \sigma}^{\rho} K_{\alpha \mu}^{\sigma} .
\end{aligned}
$$

Observe that since the connection $D$ is arbitrary, its Ricci tensor will be not be symmetric in general. Then, since the Ricci tensor $\stackrel{\circ}{R}_{\mu \alpha}$ of $\stackrel{\circ}{D}$ is necessarily symmetric, we can split Eq.(87) into:

$$
R_{[\mu \alpha]}=J_{[\mu \alpha]}, \quad R_{(\mu \alpha)}=\stackrel{\circ}{R}_{(\mu \alpha)}+J_{(\mu \alpha)} .
$$

\section{Some Important Identities}

Let $(M, g)$ be a manifold and a Lorentzian metric as defined in Section 1. Let moreover $\Lambda^{p} T^{*} M(p=0,1,2,3,4)$ be the bundle of homogeneous $p$-form fields and $\Lambda T^{*} M=\oplus_{p=0}^{4} \Lambda^{p} T^{*} M$ the bundle of non homogeneous forms fields. We define in $T^{*} M$ a metric field $\mathrm{g} \in \sec T_{0}^{2} M$ such that concerning the general bases $\left\{\boldsymbol{e}_{\mu}\right\}$ and $\left\{\theta^{\mu}\right\}$ introduced in Section 1, if $\boldsymbol{g}=g_{\mu \nu} \theta^{\mu} \otimes \theta^{\nu}$ and $\mathbf{g}=g^{\mu \nu} \boldsymbol{e}_{\mu} \otimes \mathbf{e}_{\nu}$ then $g^{\mu \alpha} g_{\alpha \nu}=\delta_{\nu}^{\mu}$. In $\Lambda T^{*} M$ we introduce a scalar product

$$
\cdot: \Lambda T^{*} M \times \Lambda T^{*} M \rightarrow \Lambda T^{*} M
$$

such that if $A, B \in \sec \Lambda^{r} T^{*} M$ are simple homogeneous $r$-forms with $A=u_{1} \wedge \cdots \wedge u_{r}$ and $B=v_{1} \wedge \cdots \wedge v_{r}$, $u_{i}, v_{j} \in \sec \Lambda^{1} T^{*} M$ then $A \cdot B=\operatorname{det}\left(\mathrm{g}\left(u_{i}, v_{j}\right)\right)$, where $\left(\mathrm{g}\left(u_{i}, v_{j}\right)\right)$ means the matrix with entries $\left(\mathrm{g}\left(u_{i}, v_{j}\right)\right)$. This scalar product is then extended by linearity and orthogonality to all $\Lambda T^{*} M$, and $A \cdot B=0$ if $A \in \sec \Lambda^{r} T^{*} M$, and $B \in \sec \Lambda^{s} T^{*} M$ with $r \neq s$. Also, if $a, b \in \sec \Lambda^{0} T^{*} M$ then $a \cdot b=a b$, the product of functions.

If the metric manifold $(M, g)$ is also endowed with an orientation, i.e., a volume 4-vector $\tau_{\mathrm{g}} \in \Lambda^{4} T^{*} M$ such that $\tau_{\mathrm{g}} \cdot \tau_{\mathrm{g}}=-1$, then a natural isomorphism between sections of $\Lambda^{r} T^{*} M$ and $\Lambda^{4-r} T^{*} M(r=0, \ldots, 4)$ can be introduced. The Hodge star operator (or Hodge dual) is the linear mapping $\underset{\mathrm{g}}{\star}: \sec \Lambda^{r} T^{*} M \rightarrow \sec \Lambda^{4-r} T^{*} M$ implicitly defined by

$$
A \wedge \underset{\mathrm{g}}{\star B}=(A \cdot B) \tau_{\mathrm{g}}
$$

for every $A, B \in \Lambda^{r} T^{*} M$. Of course, this operator is naturally extended to an isomorphism $\underset{\mathrm{g}}{\star}: \sec \Lambda T^{*} M \rightarrow$ $\sec \Lambda T^{*} M$ by linearity. The inverse ${ }_{\mathrm{g}}^{-1}: \sec \Lambda^{r} T^{*} M \rightarrow \sec \Lambda^{4-n} T^{*} M$ of the Hodge star operator is given by

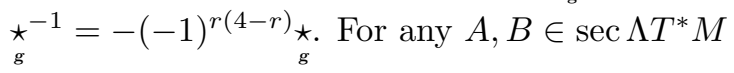

$$
A \cdot B=\langle\tilde{A} B\rangle_{0}=\langle A \tilde{B}\rangle_{0}=B \cdot A,
$$

where $\tilde{A}$ means the reverse of $A$. If $A=u_{1} \wedge \cdots \wedge u_{r}$ then $\tilde{A}=u_{r} \wedge \cdots \wedge u_{1}$ and \langle\rangle$_{0}: \sec \Lambda T^{*} M \rightarrow \sec \Lambda^{0} T^{*} M$ is the projection of a general non homogeneous form into the $\Lambda^{0} T^{*} M$ part. 
Remark 13 Suppose that $\left\{\varepsilon_{i}\right\}$ is an orthonormal basis of $\Lambda^{1} T^{*} M$ and $\left\{\varepsilon^{j}\right\}$ is reciprocal basis, i.e., $\varepsilon_{i} \cdot \varepsilon^{k}=\delta_{i}^{k}$. Then, any $Y \in \sec \Lambda^{p} T^{*} M$ can be written as

$$
Y=\frac{1}{p !} Y^{j_{1} \ldots j_{p}} \varepsilon_{j_{1}} \wedge \cdots \wedge \varepsilon_{j_{p}}=\frac{1}{p !} Y_{j_{1} \cdots j_{p}} \varepsilon^{j_{1}} \wedge \cdots \wedge \varepsilon^{j_{p}} .
$$

and

$$
Y^{j_{1} \ldots j_{p}}=Y \cdot\left(\varepsilon^{j_{1}} \wedge \cdots \wedge \varepsilon^{j_{p}}\right), \quad \quad Y_{j_{1} \ldots j_{p}}=Y \cdot\left(\varepsilon_{j_{1}} \wedge \cdots \wedge \varepsilon_{j_{p}}\right) .
$$

We define the right and left contractions of non homogeneous differential forms as follows. For arbitrary multiforms $X, Y, Z \in \sec \Lambda T^{*} M$, the left $\underset{\mathbf{g}}{(\lrcorner)}$ and right $\underset{\mathbf{g}}{(\llcorner)}$ contractions of $X$ and $Y$ are the mappings $\underset{\mathrm{g}}{\lrcorner}$ : $\sec \Lambda T^{*} M \times \sec \Lambda T^{*} M \rightarrow \sec \Lambda T^{*} M$ and $\underset{\mathrm{g}}{\llcorner}: \sec \Lambda T^{*} M \times \sec \Lambda T^{*} M \rightarrow \sec \Lambda T^{*} M$ such that

$$
(X\lrcorner Y) \cdot Z=Y \cdot(\tilde{X} \wedge Z), \quad(X\llcorner\mathrm{~g} Y) \cdot Z=X \cdot(Z \wedge \tilde{Y}) .
$$

These contracted products $\underset{g}{\lrcorner}$ and $\underset{g}{\llcorner}$ are inner derivations on $\Lambda T^{*} M$. Sometimes the contractions are called interior products. Both contract products satisfy the left and right distributive laws but they are not associative. Now some important properties of the contractions used in the calculations of the text are presented:

(i) For any $a, b \in \sec \Lambda^{0} T^{*} M$, and $Y \in \sec \Lambda T^{*} M$

$$
a \underset{\mathrm{g}}{\lrcorner} b=a\left\llcorner b=a b \text { (product of functions), } \underset{\mathrm{g}}{a}{ }_{\mathrm{g}} Y=\underset{\mathrm{g}}{\llcorner} a=a Y\right. \text { (multiplication by scalars). }
$$

(ii) If $a, b_{1}, \ldots, b_{k} \in \sec \Lambda T^{*} M$ then $\underset{a}{\lrcorner}\left(b_{1} \wedge \cdots \wedge b_{k}\right)=\sum_{j=1}^{k}(-1)^{j+1}\left(a \cdot b_{j}\right) b_{1} \wedge \cdots \wedge \check{b}_{j} \wedge \cdots \wedge b_{k}$, where the symbol $\check{b}_{j}$ means that the $b_{j}$ factor does not appear in the $j$-term of the sum.

(iii) For any $Y_{j} \in \sec \Lambda^{j} T^{*} M$ and $Y_{k} \in \sec \Lambda^{k} T^{*} M$ with $j \leq k$

$$
\left.Y_{j}\right\lrcorner Y_{k}=(-1)^{j(k-j)} Y_{k}\left\llcorner Y_{j} .\right.
$$

(iv) For any $Y_{j} \in \sec \Lambda^{j} T^{*} M$ and $Y_{k} \in \sec \Lambda^{k} T^{*} M$

$$
\left.Y_{j}\right\lrcorner Y_{k}=0, \text { if } j>k, \quad Y_{j}\left\llcorner Y_{k}=0, \text { if } j<k .\right.
$$

(v) For any $X_{k}, Y_{k} \in \sec \Lambda^{k} T^{*} M$, then $\left.X_{k}\right\lrcorner Y_{k}=Y_{k}\left\llcorner Y_{k}=\tilde{X}_{k} \cdot Y_{k}=X_{k} \cdot \tilde{Y}_{k}\right.$.

(vi) For any $v \in \sec \Lambda^{1} T^{*} M$ and $X, Y \in \sec \Lambda T^{*} M$, then $\left.\left.\left.v\right\lrcorner \underset{g}{ }(X \wedge Y)=(v\lrcorner X\right) \wedge Y+\hat{X} \wedge(v\lrcorner Y\right)$. Also, if $A, B \in \sec \Lambda^{k} T^{*} M$ then $\left.A_{\mathfrak{g}}(B\lrcorner C\right)=(A \wedge B) \underset{\mathrm{g}}{\lrcorner} C$, and $\underset{\mathrm{g}}{A\llcorner}(B\llcorner\mathrm{~g} C)=\underset{\mathrm{g}}{A\llcorner}(B \wedge C)$.

(vii) if $A, B \in \sec \Lambda T^{*} M$ then

$$
\left(A_{\mathrm{g}} B\right) \cdot C=B \cdot(\tilde{A} \wedge C), \quad\left(B_{g}\llcorner A) \cdot C=B \cdot(C \wedge \tilde{A}) .\right.
$$

Finally we present some important identities involving contractions and the Hodge dual. Let $A_{r} \in \sec \Lambda^{r} T^{*} M$ and $B_{s} \in \sec \Lambda^{s} T^{*} M, r, s \geq 0$ :

$$
\begin{aligned}
& A_{r} \wedge \underset{\mathrm{g}}{\star} B_{s}=B_{s} \wedge \underset{\mathrm{g}}{\star} A_{r} \quad r=s ; \quad A_{r} \cdot \underset{\mathrm{g}}{\star} B_{s}=B_{s} \cdot \underset{\mathrm{g}}{\star} A_{r} ; \quad r+s=n,
\end{aligned}
$$

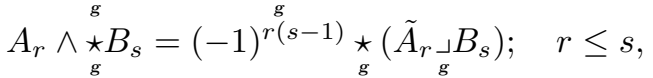

$$
\begin{aligned}
& A_{r} \underset{\mathrm{g} g}{\stackrel{\mathrm{g}}{\mathrm{g}}} B_{s}=(-1)^{r s} \underset{\mathrm{g}}{\star}\left(\tilde{A}_{r}^{\mathrm{g}} \wedge \stackrel{\mathrm{g}}{A_{s}}\right) ; \quad r+s \leq n,
\end{aligned}
$$

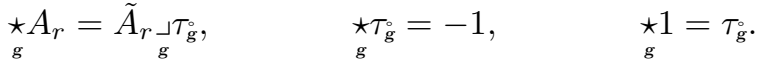




\section{References}

[1] Argurio, R. and Dehouck, F., Why not a di-NUT? or Gravitational Duality and Rotating Solutions [arXiv:0909.0542 1 [hep-th]].

[2] Argurio, R., Dehouck, F., and Houart, L., Supersymmetry and Gravitational Duality, Phys. Rev. D 79, 125001 (2009) [arXiv:0810.4999 3 [hep-th]].

[3] Barnich, G. and Troessaert, C., Manifest spin 2 duality with electric and magnetic sources, JHEP 0901:030 (2009). [arXiv:0812.0552 $2[$ hep-th]].

[4] Been, I. M. and Tucker, R. W., An Introduction to Spinors and Geometry, Adam Hilger, Bristol and New York, 1987.

[5] Bergshoeff, E. A., de Roo, M., Kerstan, S. F., Kleinschmidt, A., and Riccioni, F., Dual Gravity and Matter, Gen. Rel. Grav.41, 39-48 (2009) [arXiv:0803.1963v2 [hep-th]].

[6] Bergshoeff, E. A., Hartong J., Hohm, O. , Huebscher, M., and Ortin T., Gauge Theories, Duality Relations and the Tensor Hierarchy (in publication in JHEP)) [arXiv:0901.2054 $\mathrm{v} 2$ [hep-th] ].

[7] Bunster, C., Cnockaert S., Henneaux, M., and Portugues, R., Monopoles for Gravitation and for Higher Spin Fields, Phys. Rev.D 73105014 (2006) [arXiv:hep-th/0601222 3 ]

[8] Choquet-Bruhat, Y., DeWitt-Morette, C. and Dillard-Bleick, M., Analysis, Manifolds and Physics (revised edition), North Holland Publ. Co., Amsterdam, 1982.

[9] Dowker, J. S. and Roche, J. A., The Gravitational Analogues of Magnetic Monopoles, Proc. Phys. Soc. 92, 1-8 (1967).

[10] Fernández, V. V. and Rodrigues, W. A. Jr., Gravitation as a Plastic Distortion of the Lorentz Vacuum arXiv:0909.4472 v2 [math-ph]].

[11] Forder, P. W., Gravitomagnetic Poles and the Quantization of Frequency, Class. Quantum Grav 4, 703-710 (1987).

[12] Garcia-Compean H., Nieto, A., Obregon, O., and Ramirez, C., Dual Description of Supergravity MacDowell-Mansouri Theory, Phys. Rev. D 59, 124003 (1999) [arXiv:hep-th/9812175 v2].

[13] Garcia-Compean, H., Obregon O., Plebanski J. F., and Ramirez C., Towards a Gravitational Analog to S-duality in Nonabelian Gauge Theories, Phys. Rev. D 57, 7501-7506 (1998) [arXiv:hep-th/9711115 s3].

[14] Hehl, F. W., von der Heyde, P., Kerlick, G. D., and Nester, J. M., General Relativity with Spin and Torsion:Foundations and Prospects, Rev. Mod. Phys. 48, 393-416 (1976).

[15] Maia, A. Jr., Recami, E., Rodrigues, W. A. Jr., andRosa, M. A. F. Magnetic Monopoles without String in the Kähler Clifford Algebra Bundle: A Geometrical Interpretation, J. Math. Phys. 31, 502-505 (1990).

[16] Maia, A. Jr., and Rodrigues, W. A. Jr., A Generalized Dirac's Quantization Condition for Phenomenological Nonabelian Magnetic Monopoles, Mod. Phys. Lett A 9, 81-88 (1994).

[17] Mignani, R., Gravitational Analogues of Magnetic Monopoles from the Path-Dependent Formalism, Lett. N. Cimento 22, 597-600 (1978).

[18] Mignani, R., Path-dependent Approach to Gravitational Monopoles ,N. Cimento 56, 201-209 (1980).

[19] Nieto J. A., S-Duality for Linearized Gravity, Phys. Lett. A 262, 274-281 (1999) [arXiv:hep-th/9910049 1].

[20] Nieto, J. A., Soccoro, J., and Obregon, O., Gauge Theory of Supergravity based on a Self-dual Spin Connection, Phys. Rev. Lett. 76, 3482-3485 (1996).

[21] Quevedo F., Duality and Global Symmetries, Nucl.Phys.Proc.Suppl. A 61, 23-41 (1998) [arXiv:hep-th/9706210 1].

[22] Sachs, R. K., and Wu, H., General Relativity for Mathematicians, Springer-Verlag, New York 1977.

[23] Rodrigues, W. A. Jr. and Capelas de Oliveira, E., The Many Faces of Maxwell, Dirac and Eisntein Equations. A Clifford Bundle Approach, Springer, Heidelberg, 2007. 
[24] Rodrigues, W. A. Jr., Differential Forms on Riemann (Lorentzian) an Riemann-Cartan Structures and Some Applications to Physics, Ann. Fond. L. de Broglie 32 (special issue dedicated to torsion), 425-478 (2007) [arXiv:0712.3067v6 [math-ph]].

[25] Rosa, M. A. F. and Rodrigues, W. A., Jr.,A Geometrical Theory of Non Topological Magnetic Monopoles Mod. Phys. Lett. A 4, 175184 (1989).

[26] Witten, E., On S-Duality in Abelian Gauge Theory, Selecta Math. 1, 383-410 (1995) [arXiv:hep-th/9505186 1 1]. 